This item was submitted to Loughborough's Research Repository by the author.

Items in Figshare are protected by copyright, with all rights reserved, unless otherwise indicated.

\title{
On the efficient frequency estimation of a single tone
}

PLEASE CITE THE PUBLISHED VERSION

PUBLISHER

(C) IEEE

VERSION

VoR (Version of Record)

LICENCE

CC BY-NC-ND 4.0

REPOSITORY RECORD

Zhang, Zhuo, Andreas Jakobsson, M.D. Macleod, and Jonathon Chambers. 2019. "On the Efficient Frequency Estimation of a Single Tone". figshare. https://hdl.handle.net/2134/5611. 
This item was submitted to Loughborough's Institutional Repository (https://dspace.lboro.ac.uk/) by the author and is made available under the following Creative Commons Licence conditions.

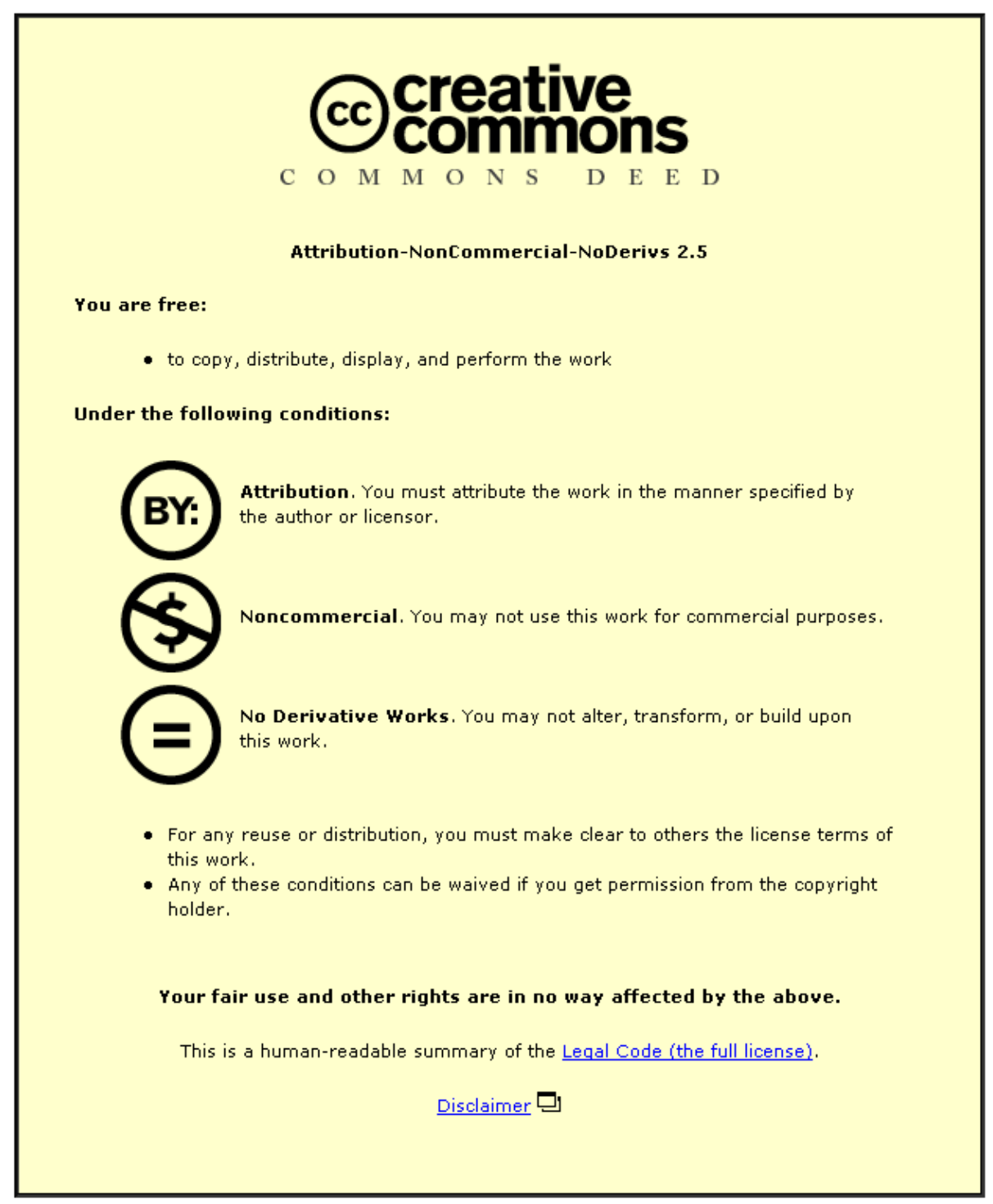

For the full text of this licence, please go to: http://creativecommons.org/licenses/by-nc-nd/2.5/ 


\title{
ON THE EFFICIENT FREQUENCY ESTIMATION OF A SINGLE TONE
}

\author{
Zhuo Zhang ${ }^{\dagger}$, Andreas Jakobsson ${ }^{\ddagger}$, Malcolm D. Macleod ${ }^{*}$, Jonathon A. Chambers ${ }^{\dagger}$ \\ $\dagger$ The Centre of Digital Signal Processing, Cardiff School of Engineering \\ Cardiff University, Cardiff CF24 OYF, UK. \\ ‡ Department of Electrical Engineering, Karlstad University, Sweden \\ * QinetiQ Ltd, St. Andrew's Road, Malvern, WR14 3PL, UK.
}

\begin{abstract}
The frequency estimation of a single tone corrupted by additive white Gaussian noise has received significant attention over the last decades due to its wide applicability in signal processing. In this paper, we propose a computationally fast and statistically improved hybrid single tone estimator which outperforms other recently proposed approaches, lowering the signal-to-noise ratio at which the Cramér-Rao lower bound is closely followed. Numerical simulations indicate that, in contrast to many other techniques, the performance of the hybrid estimator is essentially independent of the underlying frequency component.
\end{abstract}

\section{INTRODUCTION}

In a wide variety of application areas, such as biomedicine, communications and radar, one often encounters a need to find a low computational complexity estimate of the dominant frequency component of data which are assumed to consist of a single complex sinusoid corrupted by additive white Gaussian noise, and the topic has, as a result, attracted significant interest over the last decades (see, e.g., [1-14], and the references therein). The problem can be briefly stated as follows; consider the data sequence

$$
y(t)=\beta e^{i(\omega t+\theta)}+n(t),
$$

where $\beta \in \mathbb{R}, \omega$ and $\theta \in[0,2 \pi)$ denote the deterministic but unknown amplitude, frequency, and initial phase, respectively, of a complex sinusoid. Further, $n(t)$ is a circular zero mean complex white Gaussian noise with variance $\sigma_{n}^{2}$. Then, given the sequence $y(t)$, for $t=0, \ldots, N-1$, the problem is simply to estimate $\omega$ with a low computational complexity and statistically efficient estimator. In [2], Rife and Boorstyn derived the maximum likelihood (ML) estimator of $\omega$ and proposed a statistically efficient approximative ML approach involving both a combined coarse and fine search, requiring $\mathcal{O}(N \log N)$ operations. Since then, a variety of phase-based methods requiring only $\mathcal{O}(N)$ operations have been developed. In [3], for example, Tretter proposed a phase-based approach simplifying the problem to a linear regression on the phase. The method is based on a phase unwrapping algorithm, requiring a very high signal-to-noise ratio (SNR) $(S N R \gg 1)$, here defined as $S N R=\beta^{2} / \sigma_{n}^{2}$, to work well. Later, Kay proposed a modified version of Tretter's algorithm avoiding the use of the phase unwrapping algorithm [4]. The method, here termed Kay's weighted phase average (KWPA) estimator, can be shown to attain the Cramér-Rao lower bound (CRLB) for sufficiently high SNR, but the method is in general biased, and the SNR for which the CRLB is achieved depends on the underlying frequency $[6,8,12]$. As a result, the focus of recent contributions has mainly been aimed at reducing the SNR threshold [10], the frequency dependency of the threshold [7], or both [11,14].

In this paper, we propose a computationally fast and statistically improved method, termed the hybrid estimator, combining the ideas in $[10,11,14]$ to improve the performance further. The hybrid estimate is based on an initial coarse estimate of the unknown frequency using the uniformly weighted linear predictor (UWLP) method [1, 4]; this estimate is used to remove the frequency dependence of the SNR threshold. This SNR threshold is then further reduced via a combination of using an averaging filter, as suggested in [10], and an outlier removal scheme as proposed in [14]. Finally, a refined frequency estimate is formed along the lines proposed in $[10,11]$. The resulting hybrid estimator only requires $\mathcal{O}(N)$ operations.

\section{THE HYBRID PHASE-BASED FREQUENCY ESTIMATOR}

As suggested in [3], the data model in (1) can be written as

$$
y(t)=[1+v(t)] \beta e^{i(\omega t+\theta)},
$$

where $v(t)=\beta^{-1} n(t) e^{-i(\omega t+\theta)}$ is a complex white sequence. Let $v_{r}(t)$ and $v_{i}(t)$ denote the real and the imaginary parts of $v(t)$, respectively. Then, for high SNR,

$$
1+v(t) \approx e^{i \arctan v_{i}(t)} \approx e^{i v_{i}(t)},
$$


allowing the approximation

$$
y(t) \approx \beta e^{i \phi(t)},
$$

where $\phi(t)=\omega t+\theta+v_{i}(t)$. Most of the recent phasebased approaches exploit this approximation, allowing the phase to be approximately estimated from the difference of the adjacent phase values, i.e.,

$$
\Delta \phi(t) \triangleq \arg \left[y^{*}(t) y(t+1)\right] \approx \omega+v_{i}(t+1)-v_{i}(t),
$$

where $(\cdot)^{*}$ denotes the complex conjugate, suggesting the UWLP frequency estimator [1]

$$
\hat{\omega}_{c}=\arg \left[\frac{1}{N-1} \sum_{t=0}^{N-2} y^{*}(t) y(t+1)\right] .
$$

The UWLP estimator is unbiased, but statistically inefficient with variance $[4,8]$

$$
\operatorname{var}\left(\hat{\omega}_{c}\right)=\frac{1}{(N-1)^{2} S N R} .
$$

As suggested in [7,14], we will here use the UWLP estimate to form a downshifted signal, $y_{d}(t)$, to remove the frequency dependence of the SNR threshold, i.e.,

$$
y_{d}(t)=y(t) e^{-i \hat{\omega}_{c} t}
$$

In [10], Kim et al. proposed using a simple $K$-tap averaging filter prior to the frequency estimation as a way to reduce the SNR threshold. Such an averaging can be shown to lower the SNR threshold up to $10 \log _{10} K \mathrm{~dB}$. However, as such an averaging will severely restrict the allowed frequency range, the method in [10] is limited to signals with frequencies near zero. Herein, we note that the frequency content of the downshifted signal, $y_{d}(t)$, will satisfy such a restriction, and therefore propose to instead form an averaged signal as

$$
y_{f}(t)=\frac{1}{K} \sum_{k=0}^{K-1} y_{d}(t+k)
$$

Similar to (5), the adjacent phase difference of (9) can be formed as

$$
\Delta \phi_{f}(t)=\arg \left[y_{f}^{*}(t) y_{f}(t+1)\right]=\omega_{f}+u_{c}(t),
$$

where $u_{c}(t)$ is given by (23) for a general $K$. It is worth noting that the noise process $u_{c}(t)$ will now be coloured due to the average filtering [13].

As shown in [5], the SNR threshold behavior of the phase-based frequency estimators is affected by cumulative $\pm 2 \pi$ phase errors resulting from the effect of the additive noise. This effect can be countered for by introducing an outlier detection scheme. Recently, an effective scheme

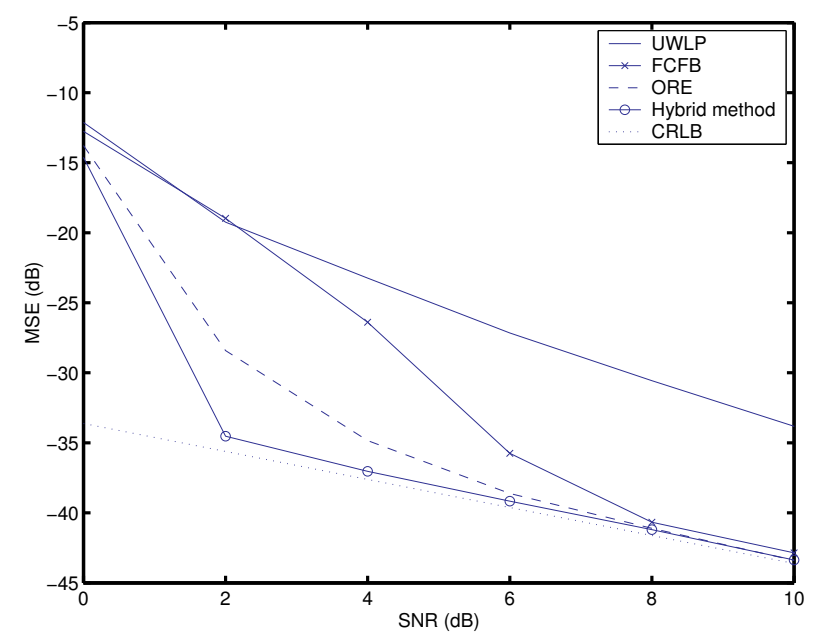

Figure 1: The MSE of the examined estimators as a function of the SNR.

was proposed in [14], where $\pm 2 \pi$ outliers are detected if $\left|\beta_{t}\right|>\left|\beta_{t-1}\right|,\left|\beta_{t}\right|>\left|\beta_{t+1}\right|$ and $\left|\beta_{t}\right|>\lambda$, with

$$
\beta_{t}=\Delta \phi_{f}(t-1)+\Delta \phi_{f}(t)+\Delta \phi_{f}(t+1),
$$

where $\beta_{-1}=\beta_{N-K}=0$ and $\Delta \phi_{f}(-1)=\Delta \phi_{f}(N-K)=$ 0 . Thus, the outliers can be removed as follows

$$
\Delta \tilde{\phi}_{f}(t)= \begin{cases}\Delta \phi_{f}(t)-\operatorname{sign}\left(\beta_{t}\right) 2 \pi & \text { if outlier detected } \\ \Delta \phi_{f}(t) & \text { otherwise }\end{cases}
$$

for $t=0, \ldots, N-K-1$. Here, $\lambda$ is a user parameter; in the simulations below, we use $\lambda=4$ (see [15] for a further discussion on the choice of $\lambda$ ).

After the SNR threshold reduction using (10) and (12), further improvement can be achieved by taking into account the colouration of the noise term in (10). This can be achieved using the FCFB approach suggested in $[11]^{1}$, whereby the frequency correction term, $\hat{\omega}_{f}$, can be found as

$$
\hat{\omega}_{f}=\sum_{t=1}^{(N-K) / K} q(t) \sum_{m=1}^{K-1} \Delta \tilde{\phi}_{f}(t K-m)
$$

where

$$
q(t)=\frac{6 t K(N-t K)}{N^{3}-N K^{2}},
$$

with $t=1,2, \ldots,(N-K) / K$. Combined with the coarse estimate, the hybrid frequency estimate is found as

$$
\hat{\omega}_{h}=\hat{\omega}_{c}+\hat{\omega}_{f}
$$

\footnotetext{
${ }^{1}$ It is worth noting that the FCFB applies a different set of weights than those used in the KWPA approach.
} 


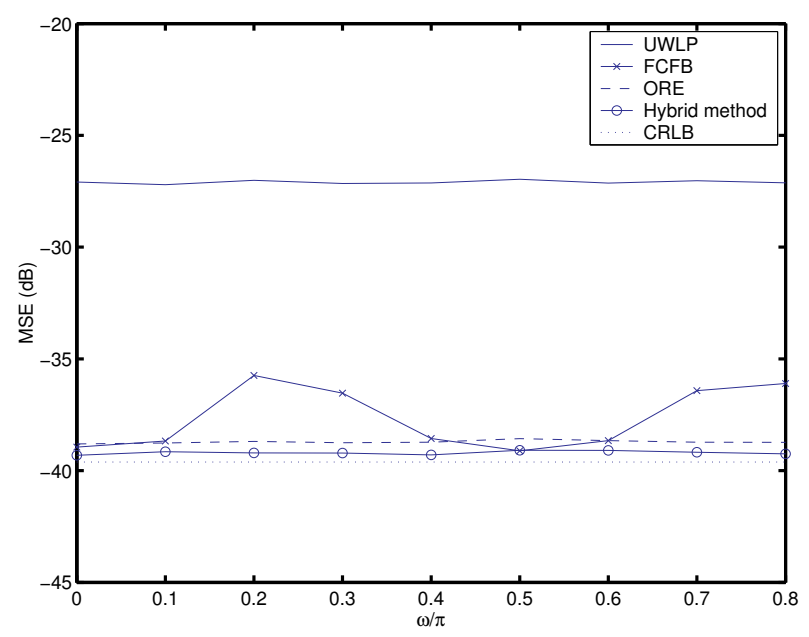

Figure 2: The MSE of the examined estimators as a function of the underlying frequency, for $\mathrm{SNR}=6 \mathrm{~dB}$.

In summary, the proposed hybrid estimator is found by first forming the downshifted signal in (8) using the UWLP estimate in (6). Then, the phase difference of the filtered signal is formed using (10), followed by the outlier removal scheme in (12). Finally, the refined frequency estimate is formed as (15), using (13). It is worth stressing that the hybrid method differs from previously suggested approaches in that it combines all the above steps; the FCFB method does not include the outlier removal scheme in (12). Similarly, the method proposed in [14], hereafter termed the outlier removal estimator (ORE), does not include the filtering in (10).

\section{SIMULATIONS}

In this section, we will briefly examine the performance of the proposed estimator. Initially, we consider $N=24$ data samples containing a single complex sinusoid with frequency $\omega=0.75 \pi$, and examine the estimated mean square error (MSE) as a function of the SNR. Figure 1 illustrates the MSE for the proposed hybrid estimator, using $K=6$, as compared to the UWLP approach [1], the FCFB approach following [11], the ORE approach [14], and the corresponding CRLB as given in [2]. As is clear from the figure, the performance of the proposed hybrid estimator is statistically improved, closely following the CRLB at a lower SNR threshold than the other examined methods. Further, the hybrid method uniformly yields a lower MSE than the other methods. It is worth noting that the hybrid estimator will suffer some performance degradation due to the introduced averaging in (9), as pointed out in [10]. This explains why the MSE of the proposed hybrid estimator can not exactly reach the CRLB as shown in Figure 1 and other figures. As

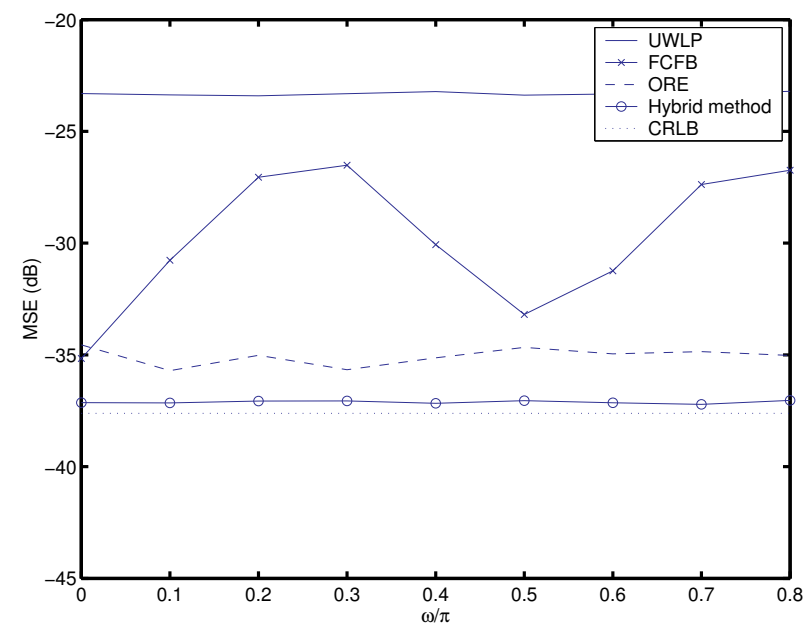

Figure 3: The MSE of the examined estimators as a function of the underlying frequency, for $\mathrm{SNR}=4 \mathrm{~dB}$.

is well known, the performance of single frequency estimators is often affected by the underlying frequency. Figures 2 and 3 illustrate how the MSE varies as a function of the frequency of the sinusoid, $\omega$, for $S N R=6 \mathrm{~dB}$ and $S N R=4$ $\mathrm{dB}$, respectively. As seen in the figures, the hybrid estimator is uniformly achieving a lower MSE than the other approaches, and is essentially independent of the underlying frequency. It is clear from the figures that the FCFB approach is significantly affected by the underlying frequency, whereas the ORE approach is showing a similar robustness as the hybrid approach, although with a somewhat worse performance. All the simulation results in the paper have been obtained using $10^{4}$ Monte-Carlo simulations. Finally, we remark that further simulations can be found in [15].

\section{APPENDIX}

In this appendix, we derive an expression for the noise process $u_{c}(t)$, given in (10), for a general $K$. Let $\omega_{f}=\omega-\hat{\omega}_{c}$. Then, using (2), $y_{f}(t)$ can be expressed as

$$
y_{f}(t)=\frac{\beta}{K} e^{i \omega_{f} t+i \theta} \sum_{k=0}^{K-1}[1+v(t+k)] e^{i \omega_{f} k}
$$

for $t=0, \ldots, N-K$. Introduce

$$
\begin{aligned}
\Psi_{K} & \triangleq \sum_{k=0}^{K-1} e^{i(k K-1) \omega_{f} / K} \\
\Phi_{v}^{K}(t) & \triangleq \sum_{k=0}^{K-1} v(t+k) e^{i(k K-1) \omega_{f} / K} .
\end{aligned}
$$


Then,

$$
\begin{aligned}
y_{f}(t) & =\frac{\beta}{K} e^{i \omega_{f} t+i \theta+i \omega_{f} / K}\left[\Psi_{K}+\Phi_{v}^{K}(t)\right] \\
& =\frac{\beta \Psi_{K}}{K} e^{i \omega_{f} t+i \theta+i \omega_{f} / K}\left[1+\Psi_{K}^{-1} \Phi_{v}^{K}(t)\right]
\end{aligned}
$$

Thus, the argument of $y_{f}(t)$ can be expressed as

$$
\begin{aligned}
\arg \left[y_{f}(t)\right]= & \arg \left[\Psi_{K}\right]+\omega_{f} t+\theta+\omega_{f} / K \\
& +\arg \left[1+\Psi_{K}^{-1} \Phi_{v}^{K}(t)\right],
\end{aligned}
$$

implying that the phase difference from adjacent samples, $\Delta \phi_{f}(t)$, can be expressed as

$$
\Delta \phi_{f}(t)=\omega_{f}+u_{c}(t)
$$

where, for $t=0, \ldots, N-K-1$,

$$
\begin{aligned}
u_{c}(t) \triangleq & \arg \left[1+\Psi_{K}^{-1} \Phi_{v}^{K}(t+1)\right] \\
& -\arg \left[1+\Psi_{K}^{-1} \Phi_{v}^{K}(t)\right]
\end{aligned}
$$

Then, using the approximation in (3), (20) can for high SNR be approximated as

$$
u_{c}(t) \approx \operatorname{Im}\left\{\Psi_{K}^{-1}\left[\Phi_{v}^{K}(t+1)-\Phi_{v}^{K}(t)\right]\right\},
$$

where $\operatorname{Im}\{x\}$ denotes the imaginary part of $x$. We note that, using a first-order Taylor expansion,

$$
\Phi_{v}^{K}(t) \approx \sum_{k=0}^{K-1} v(t+k),
$$

as $\omega_{f}$ is small due to the downshifting, implying that

$$
u_{c}(t) \approx \operatorname{Im}\left\{\Psi_{K}^{-1}[v(t+K)-v(t)]\right\} .
$$

We note that for $K=2$, (23) yields the expression given in [10], i.e.,

$$
u_{c}(t) \approx \frac{v_{i}(t+2)-v_{i}(t)}{2 \cos \left(\omega_{f} / 2\right)} .
$$

\section{REFERENCES}

[1] G. W. Lank, I. S. Reed, and G. E. Pollon, "A Semicoherent Detection and Doppler Estimation Statistic," IEEE Trans. Aerospace and Electronic Systems, vol. 9, no. 2, pp. 151-165, March 1973.

[2] D. C. Rife and R. R. Boorstyn, "Single-Tone Parameter Estimation from Discrete-Time Observations," IEEE Trans. Information Theory, vol. 20, no. 5, pp. 591-598, Sept. 1974.

[3] S. A. Tretter, "Estimating the Frequency of a Noisy Sinusoid by Linear Regression," IEEE Trans. Information Theory, vol. 31, no. 6, pp. 832-835, Nov. 1985.
[4] S. Kay, "A Fast and Accurate Single Frequency Estimator," IEEE Trans. Acoustics Speech Signal Processing, vol. 37, no. 12, pp. 1987-1990, Dec. 1989.

[5] S. W. Lang and B. R. Musicus, "Frequency Estimation from Phase Differences," in Proc. ICASSP 89, Glasgow, Scotland, 1989, pp. 2140-2143.

[6] B. C. Lovell and R. C. Williamson, "The Statistical Performance of Some Instantaneous Frequency Estimators," IEEE Trans. Signal Processing, vol. 40, no. 7, pp. 1708-1723, July 1992.

[7] V. Clarkson, "Efficient Single Frequency Estimators," in The International Symposium on Signal Processing and its Applications (ISSPA92), Gold Coast, Australia, 1992, pp. 327-330.

[8] V. Clarkson, P. J. Kootsookos, and B. G. Quinn, "Analysis of the Variance Threshold of Kay's Weighted Linear Predictor Frequency Estimator," IEEE Trans. Signal Processing, vol. 42, no. 9, pp. 2370-2379, Sept. 1994.

[9] P. Händel, "On the Performance of the Weighted Linear Predictor Frequency Estimator," IEEE Trans. Signal Processing, vol. 43, no. 12, pp. 3070-3071, Dec. 1995.

[10] D. Kim, M. J. Narasimha, and D. C. Cox, "An Improved Single Frequency Estimator," IEEE Signal Processing Letters, vol. 3, no. 7, pp. 212-214, July 1996.

[11] M. L. Fowler and J. A. Johnson, "Extending the Threshold and Frequency Range for Phase-Based Frequency Estimation,” IEEE Trans. Signal Processing, vol. 2857-2863, no. 10, pp. 212-214, Oct. 1999.

[12] B. G. Quinn, “On Kay's Frequency Estimator," Journal of Time Series Analysis, vol. 21, no. 6, pp. 707712, Nov. 2000.

[13] M. L. Fowler, "Phase-Based Frequency Estimation: A Review," Digital Signal Processing, vol. 12, no. 2, pp. 590-615, Oct. 2002.

[14] M. D. Macleod, "An Improved Fast Estimator of the Frequency of a Single Complex Tone," in Sixth IMA International Conference on Mathematics in Signal Processing, Cirencester, UK, 2004, IMA, pp. 155158.

[15] Z. Zhang, A. Jakobsson, M. D. Macleod, and J. A. Chambers, "Statistically and Computationally Efficient Frequency Estimation of a Single Tone," Tech. Rep. EE-2005-02, Dept. of Electrical Engineering, Karlstad Univ., Karlstad, Sweden, February 2005. 